\title{
Large scale hydrodynamic modes of oscillations in gaseous disks of flat galaxies with a kink on rotation curves
}

https://doi.org/10.1515/astro-2018-0031

Received Nov 20, 2017; accepted Jun 01, 2018

\begin{abstract}
The properties of unstable large-scale hydrodynamic (HD) modes of oscillations in the gaseous disk of a flat galaxy with a rotation curve having a velocity jump in the inner region of the disk are numerically investigated. It is shown that some of these modes can form a regular pseudo-ring, and others can generate a spiral structure in a model galactic disk, with the rotation curve that can approximate the observed rotation curves in nearby massive spiral galaxies (such as M31, M81). The characteristic time of formation of the regular structure turns out to be $\lesssim(1-2) G Y$, the corotation is located in the region of the velocity jump (on the radius $\sim 2-4 \mathrm{kpc}$ ). The properties of the generated density waves are determined by the parameters of the velocity jump on the rotation curve (relative amplitude and steepness of the velocity profile decrease), as well as the temperature (velocity dispersion) of the gas in the disk. For the first time, it was investigated how the global HD modes and the spiral structures formed by them will change in the case of the presence of a jump in the profile of the equilibrium surface density. This model profile was set in the form of a slightly smeared falling density step. Main results were obtained for two-arm $m=2$ azimuthal harmonic.
\end{abstract}

Keywords: galaxies: spiral structure - galaxies: kinematics and dynamics: hydrodynamic modes

\section{Introduction}

Modern observational data indicate that many massive disk (spiral) galaxies have not experienced merging and strong interactions with their neighbors during the last few billion years, see, for example, (Weinzirl et al. 2009). Such galaxies (conditionally called solitary) for times from $z \sim 1$ to $z=0$ passed through a stage of slow and smooth evolution, the so-called secular evolution (Combes 2007; Kormendy \& Kennicutt 2004; Kormendy 2013) ${ }^{1}$. An important role in the evolution of such solitary galaxies is played by large-scale collective processes of formation in the galactic disk of global structures - bars, spirals and rings (Freeman 2008; van der Kruit \& Freeman 2011; Zasov \& Sil'chenko 2010; Kormendy 2013).

It is generally accepted that the formation of regular large-scale structures in the galactic disks is due to

Corresponding Author: Yury Torgashin: Institute of Astronomy, Russian Academy of Sciences, 48 Pyatnitskaya Str. 119017 Moscow, Russia; National Research Centre "Kurchatov Institute", 1, Akademika Kurchatova pl., Moscow, 123182, Russia;

Email: torgashin@inasan.ru

Turgunbek Omurkanov: Institute of Astronomy, Russian Academy of Sciences, 48 Pyatnitskaya Str. 119017 Moscow, Russia the development of a different kind of gravitational instability (Safronov 1960; Toomre 1964, 1981; Lin \& Shu 1964, 1966; Goldreich \& Lynden-Bell 1965; Polyachenko 2004). Many of the classic results are presented in monographs (Fridman \& Polyachenko 1984; Binney \& Tremaine 2008; Bertin 2014).

According to another approach, hydrodynamic unstable modes capable of forming a spiral structure can be generated in the gas disk of a galaxy with an abrupt jump in the rotation velocity (in the inner part of the disk) (Morozov 1977, 1979; Morozov et al. 1985a,b; Torgashin 1986; Fridman 1990). A.G. Morozov first discovered and previously investigated a branch of the so-called centrifugal $(C F)$ hydrodynamic instability. It was found that in the model gaseous disk of a flat galaxy with an idealized rotation curve having a rather sharp jump in the angular velocity, $C F$ instability will be quite powerful (in increments). In subsequent works it was shown that when we move to model disks with more realistic rotation profiles

\footnotetext{
1 Over the past 15 years, many publications have appeared on the basis for the scenario of secular evolution of spiral galaxies - based on numerical modeling, analysis of modern observations, etc. We here, as a rule, give references to the main reviews, in which the reader can find a detailed bibliography.
} 
that have a fairly smooth velocity jump, the instability is gradually weakened and stabilized, while no other unstable branches have been detected until recently. Partially this gap was eliminated in (Torgashin \& Omurkanov 2013), below (TO).

In the present work, the numerical study of unstable HD modes in the model gas disk of a flat galaxy with a velocity jump on the rotation curve, started in (TO), is continued. As a result, for the first time the modes in discs with the parameters and the shape of the velocity jumps actually corresponding to those observed in a number of massive disc galaxies (for example, M31 and M81) were investigated. In addition, the effect of the radial gradient of the equilibrium surface density of a gas disk on the development of unstable HD modes was studied (to the best of our knowledge, previously HD modes have been studied only in homogeneous density gaseous galactic disks). It is shown that the presence of a noticeable gradient in the surface density of a disk in the region of the velocity jump increases the chances of manifestation of unstable resonant HD branches of oscillations and the formation of a large-scale pseudo-circular structure in this region of the disk. Conclusions are made about the ability of unstable HD modes to form large-scale regular structures in the gas disks of some spiral galaxies at a certain stage of their evolution.

\section{Conditions under which unstable HD modes of oscillations in the gas disk of a flat galaxy can manifest themselves}

For the manifestation of the mechanisms of large-scale HD instabilities in the galaxy, it is necessary, firstly, to have a noticeable jump in the rotation velocity (in the inner region of the disk), with a steeper velocity decrease with a radius, than in the Keplerian law, and secondly, more powerful mechanisms of gravitational instabilities in flat subsystems were suppressed. It is assumed that such properties of the galaxy could have formed at the previous stages of its secular evolution.

As follows from many numerical experiments on the evolution of stellar galactic disks, the bar-mode formed due to collective gravitational perturbations, and/or the spiral density wave, causes gas flows from the intermediate radii to the center of the disk and to the periphery, see, for example, (Athanassoula 1992; Salo et al. 1999). As a result of star formation, this leads to the formation in the central region of the galaxy of a massive flattened condensation - pseudo-bulge. If a fairly flattened and comparatively massive stellar pseudo-bulge is formed in a massive galaxy, with a radial size $a_{\text {bulge }}$ and a fairly sharp boundary along the radius ${ }^{2}$, then the total distribution of the equilibrium gravitational potential is able to create, in the region of the disk near the radius $r \sim(1.2-1.5) a_{b u l g e}$, the fall of the rotation curve $V_{\varphi}(r)$ with a radius faster than Keplerian law:

$$
\begin{aligned}
& V_{\varphi}(r) \propto r^{\left(\alpha_{\Omega}+1\right)} ; \quad \Omega(r) \propto r^{\alpha_{\Omega}} ; \\
& \alpha_{\Omega \min } \approx(-1.7)-(-1.8) ;
\end{aligned}
$$

Another mechanism for the formation of velocity jump in the rotation curve in the galactic disk is considered in the article (Kondratyev 2014).

Rotational curves with a velocity jump are observed in a number of nearby massive galaxies, see, for example, (Sofue \& Rubin 2001; Chemin et al. 2009; Tenjes et al. 1998), and Figure 1 (M31, M81). Our preliminary studies on the construction of a self-consistent model of the large-scale mass distribution in the disk galaxy within the framework of the Einasto-Haud model (Einasto \& Haud 1989) with 5 main components (old bulge + disk, young thin pseudobulge + disk, and extended halo, the disk has a density depression at the center), show that the value of the parameter $\alpha_{\Omega \text { min }}$, that determines the fastest decay of the angular velocity with the radius, with reasonable set of the mass-model parameters, that do not contradict the observations, can reach values $\approx(-1.80)-(-1.85)$. A similar estimate was obtained earlier, see (Arakcheev \& Torgashin 2012; TO).

Another important consequence of the development in the stellar galaxy disk of a bar mode, or/and of a spiral density wave, is the effects of heating of the stellar gas noted in early numerical experiments - its temperatures (stellar velocities dispersion) increase. Modern observational data confirm these facts, showing a noticeable increase in the dispersion of the velocities of stellar subsystems with an increase in their age (determined by chemical composition), see, for example, (Holmberg et al. 2007). Such evolutionary warming up of young stellar disk subsystems means the approaching of these subsystems (according to the Safronov-Toomre criterion, and its later variants taking into account open spirals) to the boundary of gravitational instability. In many numerical exper-

2 The characteristic radial scale-size $a_{\text {bulge }}$ of such a pseudo-bulge in a massive spiral galaxy, based on the shape of the rotation curve and the model mass distribution in the galaxy, is estimated as $a_{\text {bulge }} \sim(1-2) k p c$. 


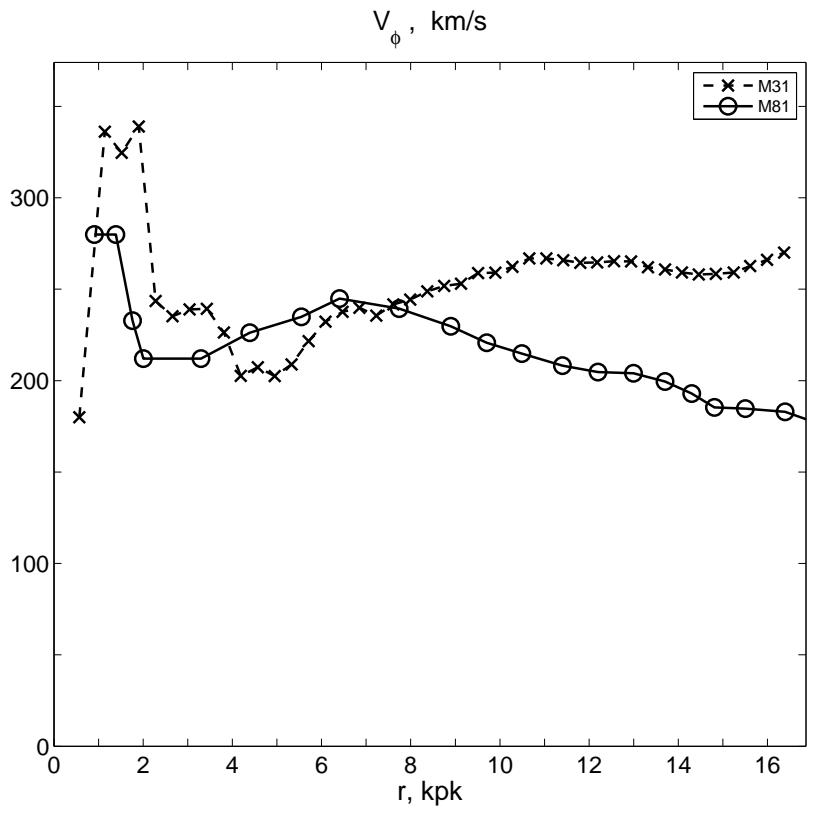

Figure 1. Observed rotation curves of galaxies having velocity jump in the inner disk region: M31 - crosses on a dashed line (Chemin et al. 2009); M81 - circles on a solid line (Tenjes et al. 1998).

iments, at later stages, the spirals, or bar mode gradually began to blur, respectively, the bar merged with the pseudo-bulge (Bournaud et al. 2005; Sellwood 2011).

The above facts and considerations speak in favor of the possibility of the situation when, at some stage of the evolution of the disk galaxy, its quasi-equilibrium state will satisfy the two required conditions. The first is the proximity to the boundary of instability with respect to the powerful gravitational instabilities (or the stabilization of these instabilities). The second is the formation of a mass distribution of large-scale components of the galaxy, in which a gravitational potential distribution is created, in which the equilibrium angular velocity of the gas disk, $\Omega(r)$, has a region with relatively steep radial decrease, see (1). In this case, conditions are created for the manifestation of large-scale, so-called gradient, hydrodynamic mechanisms of instability in a gas galactic disk (Morozov 1977, 1979; Morozov et al. 1985a,b; Fridman 1990).

We note that it is practically impossible to separate gravitational and hydrodynamic instabilities in a real galaxy, since in the equations describing the dynamics of perturbations in a gas disk, there are always terms with gradients of the perturbed gravitational potential, and terms with gradients of the perturbed pressure. Here, we are talking about the fact that the properties of HD modes are manifested in "pure form" in cases when the terms with pressure gradients dominate, although selfgravitation even in these situations may be important, but not basic in determining the properties of unstable modes of a disk. This is the situation we are exploring here, at the first stage, discarding terms with self-gravity.

\section{A brief description of the investigation of $\mathrm{HD}$ modes in a model gaseous disk with a velocity jump on the rotation curve}

First, a model disk is constructed that approximates the gas disk of a flat galaxy with a velocity jump on the rotation curve. For the selected galaxy, on the basis of the observed rotation curve $V_{\varphi g l x}(r)$, the values of the velocity jump parameters are settled:

$$
\left\{R_{\alpha \Omega} ; V_{\varphi \max } ; \delta V_{\varphi} ; \alpha_{\Omega \min }\right\}
$$

Here $R_{\alpha \Omega}$ - is the radius of the steepest fall of the rotation curve in the region of the velocity jump, it is approximately equal to the average radius of the velocity jump region; $V_{\varphi \max }-$ magnitude of the internal maximum of azimuthal velocity; $\delta V_{\varphi} \equiv\left(V_{\varphi \max }-V_{\varphi \min }\right) / V_{\varphi \max }$ - relative amplitude of the velocity jump; $\alpha_{\Omega \min }=\min _{r}\left(\alpha_{\Omega}(r)\right)=\alpha_{\Omega}\left(r=R_{\alpha \Omega}\right)-$ the parameter of the most rapid fall of the angular rotation velocity with a radius, where $\alpha_{\Omega}(r)=\frac{d \ln \Omega_{0}(r)}{d \ln r}$; $\Omega_{0}(r)=V_{\varphi}(r) / r$ - is the equilibrium angular rotation velocity in the gaseous disk.

Taking into account the fact that the observed $V_{\varphi g l x}(r)$ has a scatter of data related to observation errors, and also taking into account possible non-circular motions in the galactic disk (which we assume to be small), the constructed model function, $V_{\varphi}(r)$, should not only have parameters that coincide with (2). Besides that, it must satisfy the modern constructed mass distribution models for disk galaxies, see, for example, (Einasto \& Haud 1989; Tenjes et al. 1998). From the analysis of the results of these models, in particular, there are restrictions on the possible steepness of the decrease of $V_{\varphi}(r)$ profile in the region of the velocity jump, i.e. on the parameter $\alpha_{\Omega \text { min }}$, see (1).

In the case of an inhomogeneous density disk, it is necessary in the same way to additionally specify a simple model of the equilibrium surface gas density in the disk, $\sigma_{0}(r)$.

For greater universality of the results obtained, we further normalize the basic functions to given charac- 
teristic scales in the galaxy. The radii and velocities are normalized, respectively, to the values $R_{\alpha \Omega}$ and $V_{\varphi \max }$, then the frequencies will be normalized by the value $\Omega_{\text {norm }}=V_{\varphi \max } / R_{\alpha \Omega}$. The surface density is normalized to the value $\sigma_{02}$ - the unperturbed density in the outer part of the disk (relative to the velocity jump).

We use the traditional 2-D system of equations (Euler's equations of motion, the continuity equation and the adiabaticity equation of gas motion). Taking into account the equilibrium axisymmetric stationary state, after linearizing the equations, neglecting the terms with self-gravitation, then we pass to the spectral harmonics ${ }^{3}$ $\tilde{f}^{(m, \omega)}(r)$ of the perturbations.

As a result, we obtain a system of two linear homogeneous ordinary differential equations for the perturbed normalized enthalpy $\tilde{\eta}$ and the perturbed radial Lagrange' displacement $\tilde{\xi}$, see, for example, (Torgashin 1986; TO):

$$
\begin{aligned}
\frac{d \tilde{\eta}}{d r} & =\left[\frac{2 m \Omega_{0}}{\hat{\omega}}-\frac{y-1}{y} \alpha_{\sigma}\right] \frac{\tilde{\eta}}{r} \\
& +\left[\left(\hat{\omega}^{2}-\kappa^{2}\right)-\frac{y-1}{y^{2}} \frac{\alpha_{\sigma}^{2}}{M_{\star}^{2} r^{2}}\right] \tilde{\xi} ; \\
\frac{d \tilde{\xi}}{d r} & =\left[\frac{m^{2}}{r^{2} \hat{\omega}^{2}}-M_{\star}^{2}\right] \tilde{\eta}-\left[1+\frac{2 m \Omega_{0}}{\hat{\omega}}+\frac{\alpha_{\sigma}}{y}\right] \frac{\tilde{\xi}}{r} .
\end{aligned}
$$

The remaining perturbed values are expressed through $\tilde{\eta}$ and $\tilde{\xi}$ in a linear algebraic way:

$$
\begin{gathered}
\tilde{V}_{r}=-i \hat{\omega} \tilde{\xi} ; \quad \tilde{V}_{\varphi}=\frac{m}{\hat{\omega}} \frac{\tilde{\eta}}{r}-\frac{\kappa^{2}}{2 \Omega_{0}} \tilde{\xi} ; \\
\tilde{\sigma}=\sigma_{0}\left[M_{\star}^{2} \tilde{\eta}-\frac{y-1}{y} \alpha_{\sigma} \frac{\tilde{\xi}}{r}\right] .
\end{gathered}
$$

We used following designations:

$$
\begin{aligned}
\hat{\omega}(r) & =\omega-m \Omega_{0}(r) ; \quad \Omega_{0}(r)=V_{\varphi 0}(r) / r ; \\
\kappa^{2}(r) & =4 \Omega_{0}^{2}\left(1+\alpha_{\Omega} / 2\right) ; \quad \tilde{\eta}=\tilde{p} / \sigma_{0} ; \\
M_{\star} & =V_{\varphi \max } / C_{S 0} ; \quad \alpha_{f}=d \ln f_{0}(r) / d \ln r .
\end{aligned}
$$

Here $\tilde{p}$ and $\tilde{\sigma}$ - are the perturbations of the plane pressure and the surface density (the unperturbed equilibrium functions are denoted by the subscript " 0 "), $C_{S 0}$ - is the dispersion of the gas velocities (which was assumed to be equal to its value in the region of the velocity jump), $y-$ is plain adiabatic index, $m$ - is the azimuthal harmonic number. Taking into account the corresponding boundary conditions, we calculate the eigenvalues $\omega$ and eigenfunctions $\tilde{f}$ for system (3). With the aid of (4) we find the remaining perturbations.

3 The perturbations are presented in the complex form $\hat{f}(r, \varphi, t) \propto \tilde{f}^{(m, \omega)}(r) \cdot \exp [i(m \varphi-\omega t)]$.

\section{Results of the investigation of HD modes in the model gaseous galactic disk}

Recent studies of unstable HD modes in a thin non-selfgravitating model gas disk, approximating the gas galactic disk, made it possible to reveal for the first time the presence of a set of "low-frequency" $A_{k}$, and "high-frequency" $B_{j}$ unstable oscillation branches along with the known centrifugal branch of oscillations, $C F$, see (TO).

The new unstable HD branches of oscillations found in (TO) were interpreted, by analogy with the works (Fridman et al. 2006, 2008), as overreflection modes. Such modes were, apparently, first found by Kolykhalov (Kolykhalov 1984) - in a plane-parallel compressible gas flow with supersonic vortex sheet and a parallel rigid wall (providing feedback).

Investigations of unstable HD modes in (TO) were carried out for a model disk with a rotation profile given in the form:

$$
\begin{aligned}
V_{\varphi t h}(r) & =r \cdot \Omega_{t h}(r) \\
& =r \cdot\left[\langle\Omega\rangle-\frac{\Delta \Omega}{2} \cdot \tanh \left(\frac{r-R_{\Omega}}{L_{\Omega}}\right)\right] ;
\end{aligned}
$$

The parameters set $\left\{\langle\Omega\rangle ;\left(\frac{\Delta \Omega}{2}\right) ; R_{\Omega} ; L_{\Omega}\right\}$ for calculating the model $V_{\varphi t h}(r)$ was found from the condition, that the profile (6) must give the same defining parameters, as the galaxy set (2).

The equilibrium surface density was assumed constant, $\sigma_{0}(r)=$ const, $\alpha_{\sigma}=d \ln \sigma_{0} / d \ln r=0$.

The equilibrium state of the model gas disk, taking into account the normalization used, was determined by three dimensionless parameters: $\left\{\delta V_{\varphi} ; \alpha_{\Omega \min }\right\}$, describing the shape of the model rotation curve, and the gas temperature, which was determined by

$$
M_{\star}=V_{\varphi \max } / C_{S 0},
$$

see (5).

In (TO), the main unstable branches of oscillations for the most large-scale azimuthal harmonics $m=1,2,3$ were investigated, with relative amplitudes of the velocity jump $\delta V_{\varphi} \in[0.2 ; 0.45]$, in the range of parameters $\alpha_{\Omega \text { min }} \in[-6.0 ;-2.0], M_{\star} \in[1 ; 35]$. Calculations have shown that the increments of unstable modes fall significantly from idealized profiles with a sharp velocity jump, $\alpha_{\Omega \text { min }}=-6$, to profiles that are closer to the real ones, with $\alpha_{\Omega \text { min }}=-2$. Moreover, the unstable $C F$ oscillation branch, which forms a regular spiral density wave, has already stabilized when $\alpha_{\Omega \text { min }}=-2$. Use for a disk with $\alpha_{\Omega \text { min }}=-2$ 
a slightly modified (steepened in the central region of the jump) rotation profile, obtained from (6) without changing the values $\left\{R_{\alpha \Omega} ; V_{\varphi \max } ; \delta V_{\varphi} ; \alpha_{\Omega \min }\right\}$, somewhat strengthened (by increments) the weakly unstable modes, and revived $C F$ unstable branch, for more details, see (TO).

Although important results were obtained in (TO) on unstable HD modes in a gas galactic disk, certain fundamental questions remained unclear.

1. How much will the eigenvalues and eigenfunctions of unstable modes change if on the periphery of the disk the velocity profile is not described by $\Omega(r) \rightarrow$ const, but, as is the case in a real galaxy, by $V_{\varphi}(r) \rightarrow$ const, see Figures 1,2 .

2. Are the unstable branches of oscillations preserved if the steepness of the profile is a real value, $\alpha_{\Omega \min } \approx(-1.80)-(-1.85)$, see (1) and below in the text.

On the basis of some trial calculations, it was noted in (TO) that the influence of the periphery of the profile (6) on the unstable modes is rather weak, and that with an increase in the value $\alpha_{\Omega \text { min }}$ from -2 up to about -1.7 , the instability should stabilize. Nevertheless, detailed studies for a disk with more real model profiles have not been carried out, and unstable modes in the case of profiles with a parameter $\alpha_{\Omega \text { min }} \approx(-1.80)-(-1.85)$ have not been practically studied.

In this paper, we present the results of investigations that give answers to the unresolved questions mentioned above. Here, as a base profile, describing the rotation curve, is used the one, which on the periphery of the disk goes to the plateau:

$$
\begin{aligned}
& V_{\varphi \text { th tail }}(r)=r \cdot \Omega_{\text {th tail }}(r) ; \\
& \Omega_{\text {th tail }}(r)=\Omega_{\text {th }}(r) \cdot f_{\text {tail }}(r) ; \\
& f_{\text {tail }}(r)=\left[1+\left(r / R_{\text {tail }}\right)^{N}\right]^{-1 / N} ; \\
& N=\{2-6\} ;
\end{aligned}
$$

here function $\Omega_{t h}(r)$ - from the formula (6).

The results below are obtained with $N=4$. When using (7), (8), a parameter $q_{\text {tail }}=V_{\varphi \infty} / V_{\varphi \max }$ is added to the set of defining parameters of the profile, which defines the velocity value on the plateau:

$$
\left\{R_{\alpha \Omega} ; V_{\varphi \max } ; \delta V_{\varphi} ; \alpha_{\Omega \min } ; q_{\text {tail }}\right\}
$$

The main results presented below are obtained for a disk with a model rotation profile (7), (8), with the following set of defining parameters:

$$
R_{\alpha \Omega}=2.85 \mathrm{kpc} \quad V_{\varphi \max }=340 \mathrm{~km} / \mathrm{s}
$$

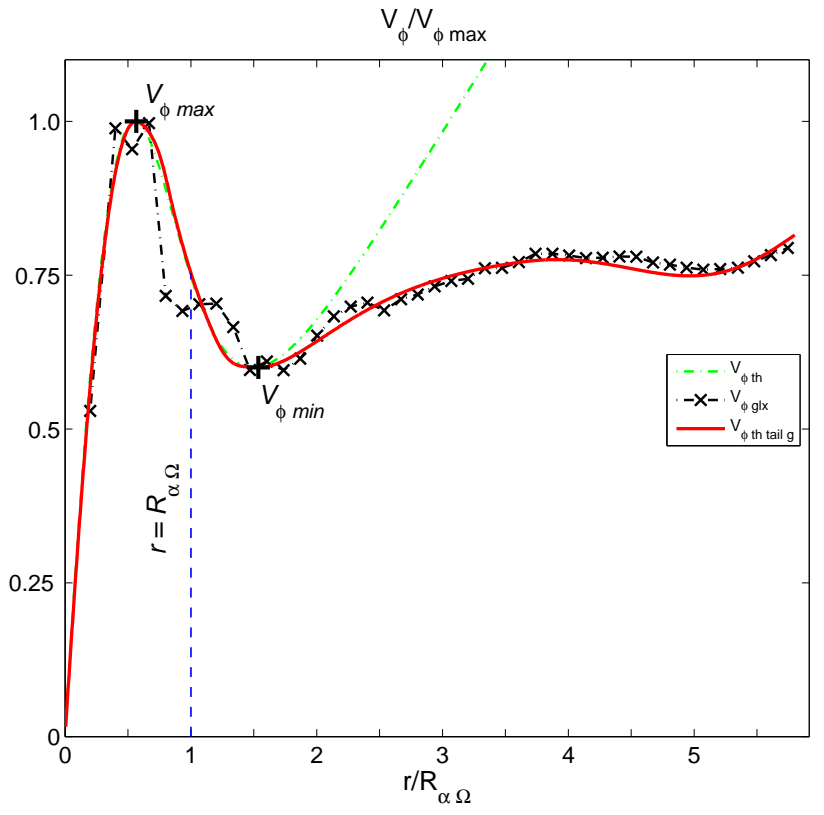

Figure 2. Rotation curves in normalized units: the same as in the Figure 1 for the $M 31$ - crosses and dashed lines; the model rotation curve (7), (8), used in this work - thick solid red line; the profile (6) used in the previous work (TO) - green dotted line; normalization parameters: $V_{\varphi \max }=340 \mathrm{~km} / \mathrm{s}, R_{\alpha \Omega}=2.85 \mathrm{kpc}$; other parameters - see text.

$$
\begin{array}{ll}
\delta V_{\varphi}=0.40 ; & \alpha_{\Omega \min }=-1.85 ; \\
q_{\text {tail }}=0.80 ; & N=4 ;
\end{array}
$$

In the central part of the velocity jump region, the velocity profile was steepened, similar to that used in (TO). As can be seen from Figure 2, with the chosen set of parameters (10), the model rotation curve satisfactorily approximates the observed rotation curve in the galaxy M31 (Chemin et al. 2009).

It is this model velocity profile that was plotted on Figure 2 by thick solid red line together with the observed rotation curve of galaxy M31, (Chemin et al. 2009). As we noted, functions on Figure 2, as well as all other functions here, were normalized, and the normalization values for velocity and radius are $V_{\varphi \max }$ and $R_{\alpha \Omega}$ respectively, see (10).

First, within the framework of the model disk with homogeneous density, the influence of the shape of the velocity profile in the outer part of the disc (relative to the velocity jump region) on the results of the simulation of the HD modes was carried out. For a model disk with the parameters given in (10), but with $\alpha_{\Omega}$ min $=-2.0$, a comparison was made of the excited modes for the cases of the rotation profile (6), and the profile (7), (8). 
The differences in the eigenvalues and eigenfunctions of the excited modes, with other fixed determining parameters, turned out to be within $\sim 3 \%$. This indirectly confirms that the excitation mechanism of unstable HD modes is determined by the "generator" - the region of the velocity jump in the disk.

In the second stage, a study was made of the changes in HD modes in a model disk with a rotation profile (7), (8), determined by set of parameters (10), when we move from $\alpha_{\Omega \text { min }}=-2.0$ (Figure 3) to a more realistic value $\alpha_{\Omega \min }=-1.85$, Figure 4 . The last rotation profile completely corresponds to both the observed galactic rotation curve and our preliminary results on the construction of a self-consistent 5-component model of the galaxy, see paragraph 1.

It turns out that a small, at first glance, change in the steepness of the profile, defined by parameter $\alpha_{\Omega \text { min }}$, significantly changes the properties of the unstable HD modes excited in the disk. It was found that not only a significant weakening of the increments of unstable modes occurs, but also the suppression of some unstable branches of oscillations, see Figures 3, 4.

The figures represent the calculated pictures of the eigenvalues - complex frequencies $\omega$, depending on the varying parameter $M_{\star}$, - for perturbations in the model disk with velocity profile (7), (8), having a set of parameters (10), for the azimuthal harmonic $m=2$. The top panel presents the phase velocities $\omega_{r} / m$, and the bottom panel - the increment $\omega_{i}$. The velocity profile steepness parameter $\alpha_{\Omega \text { min }}$ is changed in the disk, from Figure 3 to Figure 4, correspondingly from the value -2.0 to the value -1.85 .

The range of the parameter $M_{\star}$ that is most interesting for applications to the gaseous galactic disk can be estimated $\mathrm{as}^{4}: 15 \lesssim M_{\star} \lesssim 25$. It is in this range of the parameter $M_{\star}$ that the basic calculations of the eigenvalues (complex eigenfrequencies of oscillations) and eigenfunctions of unstable branches of hydrodynamic modes were performed.

When we moved from a disk with a rotation profile with $\alpha_{\Omega \min }=-2.0$, Figure 3, to a disk with a rotation profile with $\alpha_{\Omega \min }=-1.85$, Figure 4 , the $C F$ oscillation branch in the "main" range of parameter $M_{\star}$ has completely stabilized, and the additional low-frequency

4 The values of the maximum of the rotation velocity in massive spiral galaxies are usually $V_{\varphi \text { max }} \sim 250-350 \mathrm{~km} / \mathrm{s}$, and in the case we have chosen, $V_{\varphi \max } \approx 340 \mathrm{~km} / \mathrm{s}$. According to observations, in the inner region of the disk, the dispersion of the gas velocities can be estimated as $C_{S 0} \sim 12-20 \mathrm{~km} / \mathrm{s}$, see, e.g., (Haan et al. 2009). This gives a rough estimate of the range of the parameter $M$ *
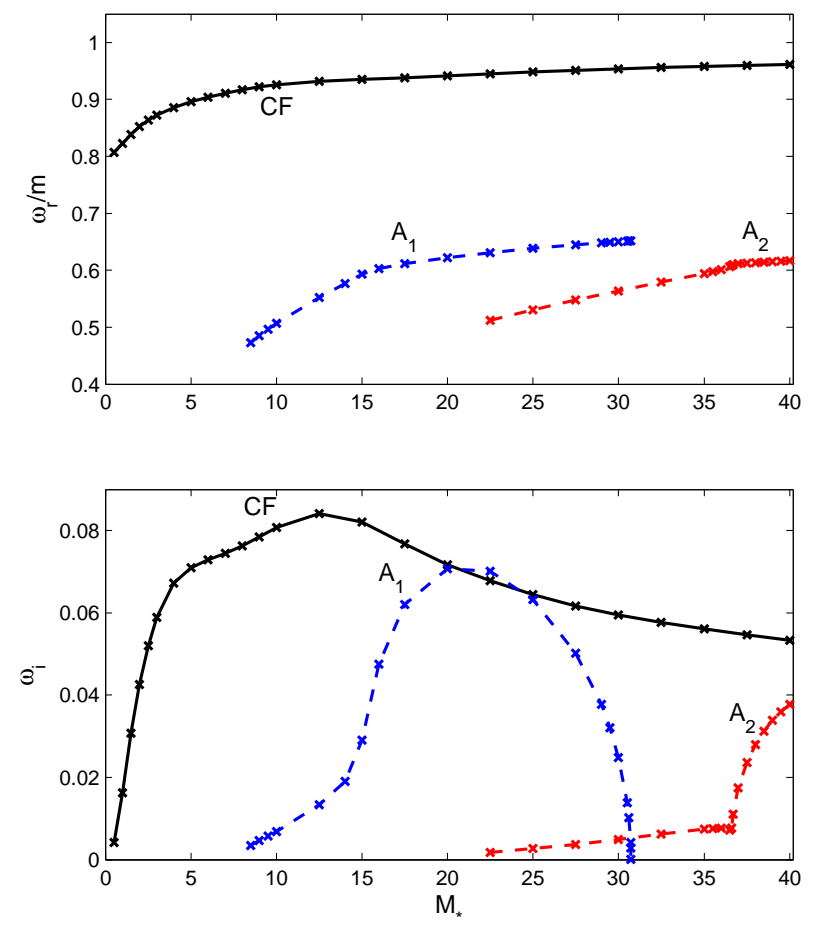

Figure 3. Calculated eigenvalues of the main unstable branches depending on the parameter $M_{\star}$ in the model gas disk, having rotation profile (7), (8) with parameters (10), but $\alpha_{\Omega \text { min }}=-2.0$. Case of uniform unperturbed surface density. Top panel - phase angular velocity of waves $\omega_{r} / m$, bottom panel - increment $\omega_{i}$.

branch $A_{0}$ is practically not relevant because of small increments and its stabilization at $M_{\star} \gtrsim 13$, see Figure 4 .

So, as it can be seen from the results shown in Figure 4, in the model disk with a uniform surface density, and with a rotation profile (7), (8), having a complete set of parameters (10), practically $A_{1}$ was the only unstable low-frequency branch that survived.

Two basic approaches can be used to analyze the spatial form of perturbations. Taking into account the complex representation of the perturbations (footnote (3)), we can write the real perturbations at a fixed time moment in the form:

$$
\begin{aligned}
\operatorname{Re}\{\hat{f}(r, \varphi)\} & =A_{f}(r) \\
& \cdot \cos \left\{m\left[\varphi-\psi_{f}(r)+\varphi_{0}\right]\right\}
\end{aligned}
$$

Here, the amplitude $A_{f}(r)$ and phase $\psi_{f}(r)$ of the perturbed function $\hat{f}(r, \varphi)$ are determined through the absolute value and the argument of the complex function $\tilde{f}(r)$ in the standard way:

$$
A_{f}(r)=|\tilde{f}(r)| ; \quad \psi_{f}(r)=-\frac{1}{m} \operatorname{Arg}\{\tilde{f}(r)\} ;
$$

The values of the initial phase $\varphi_{0}$, at $\varphi_{0}=(2 \pi / m) \cdot n$, $n=0 ; \pm 1 ; \pm 2 ; \ldots-$ correspond in (11) to perturbation 

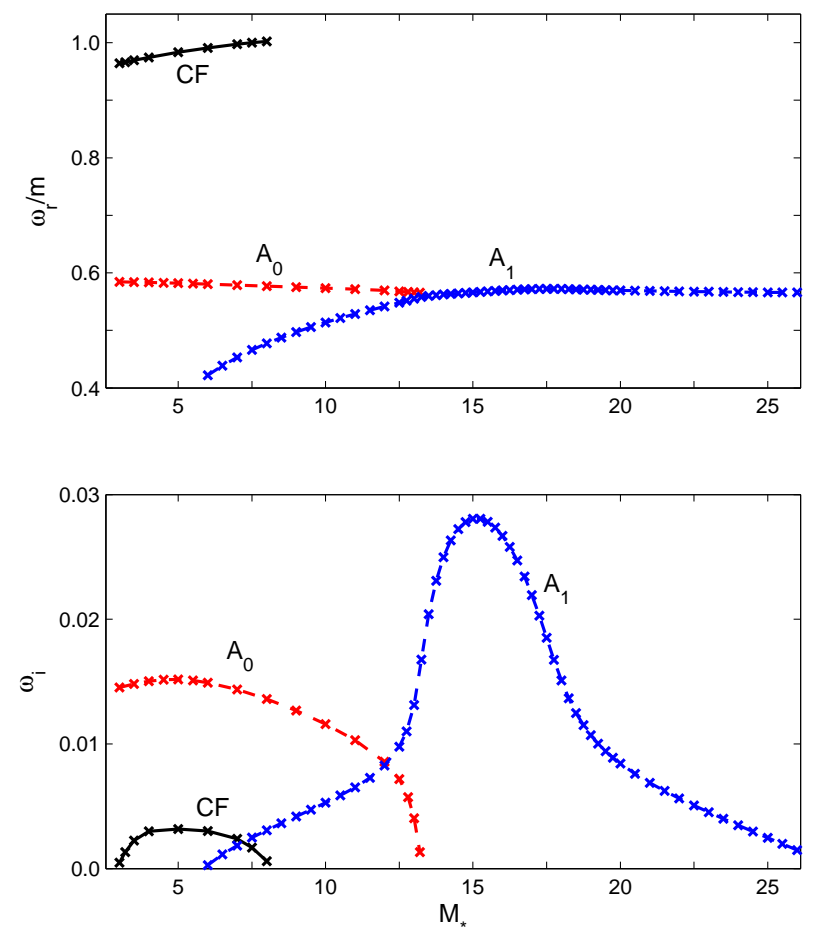

Figure 4. The eigenvalues of the main unstable branches depending on the parameter $M_{*}$, in the model gas disk as in Figure (3), but with more realistic velocity profile, having the steepness velocity jump parameter $\alpha_{\Omega \min }=-1.85$.

maxima at each radius, and $\varphi_{0}=(\pi / m) \cdot n,-$ determine the minima.

For example, the phase curve $\psi_{\sigma_{1}}(r)$ of calculated perturbed surface density $\sigma_{1}(r, \varphi)$ as the eigenfunction, corresponding to the eigenvalue $\omega$ of unstable branch $A_{1}$, azimuthal harmonic $m=2$, and parameter $M_{\star}=15.0$, is presented on Figure 5, bottom panel. The curve describes the phase $\varphi$ of the amplitude maximum of eigenfunction $\sigma_{1}(r, \varphi)$ at each radius $r$. The corresponding maximum amplitude $A_{\sigma_{1}}(r)$ is presented on the top panel of the Figure.

Another standard way of representing perturbations is the image of the surface $\operatorname{Re}\{\hat{f}(r, \varphi)\}$. On Figure 6 the same calculated perturbed surface density $\sigma_{1}(r, \varphi)$ for $A_{1}$ unstable root, as in Figure 5, is presented. Also on this Figure, the perturbed vector velocity field in the coordinate frame, rotating with the angular velocity $\Omega=\Omega_{p h}$, where $\Omega_{p h}=\omega_{r} / m$ - is the phase angular velocity of the root $A_{1}$, - is shown. As is known, perturbed vector velocity field (in co-rotating frame) of HD modes in the considered case, forms an anticyclonic vortexes, with centers near corotation circle (TO). These centers on Figure 6 denoted by red stars.
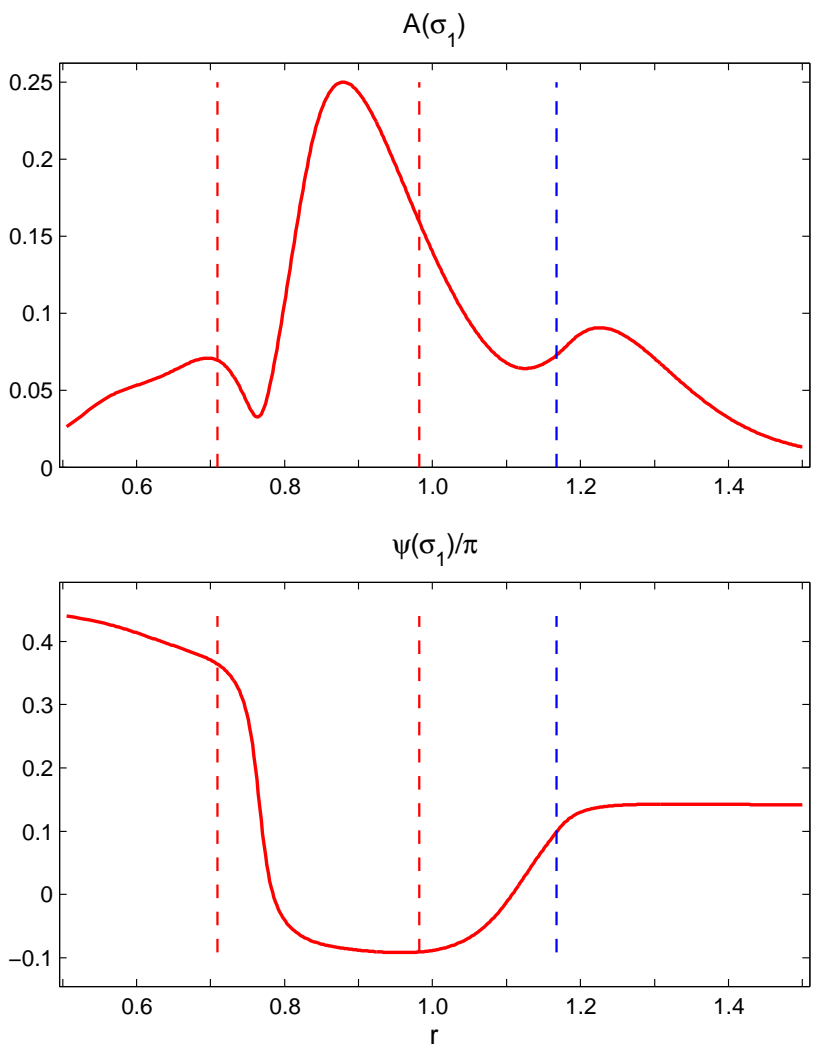

Figure 5. Amplitude (top) and phase (bottom) of the perturbed surface density $\sigma_{1}(r, \varphi)$ for eigenfunction, corresponds to the eigenvalue $\omega$ of unstable branch $A_{1}$, azimuthal harmonic $m=2$, see Figure 4, with fixed value of parameter $M_{*}=15.0$. The model disk has uniform unperturbed surface density, and rotation profile (7), (8), with parameters (10). Here the annular radial interval encompassing the region of the velocity jump is represented. Vertical dashed lines show the radii of resonances: blue - corotation, red - Lindblad's. In the shown here annular region, there are two internal Lindblad resonances, the outer one is somewhat further along the radius.

The unstable HD modes of oscillations of different branches are large-scale gravitational-acoustic growing waves in an inhomogeneous (along the radius) gas galactic disk.

In particular, perturbations for unstable lowfrequency oscillation branches have an internal "wave zone" (relative to their corotation radius located inside the velocity jump, closer to its periphery). We can see it on figures 5 and 6 , where the perturbed surface density $\sigma_{1}(r, \varphi)$ of branch $A_{1}$, azimuthal harmonic $m=2$, is shown. From figure 6 it is clear, that the perturbations of surface density for a branch $A_{1}$, harmonic $m=2$, can be represented as two tightly wound quarter-turn spirals localized along radius near the corotation circle. So, this unstable HD mode can form an analog of inhomogeneous and not quite circular pseudo-ring. 


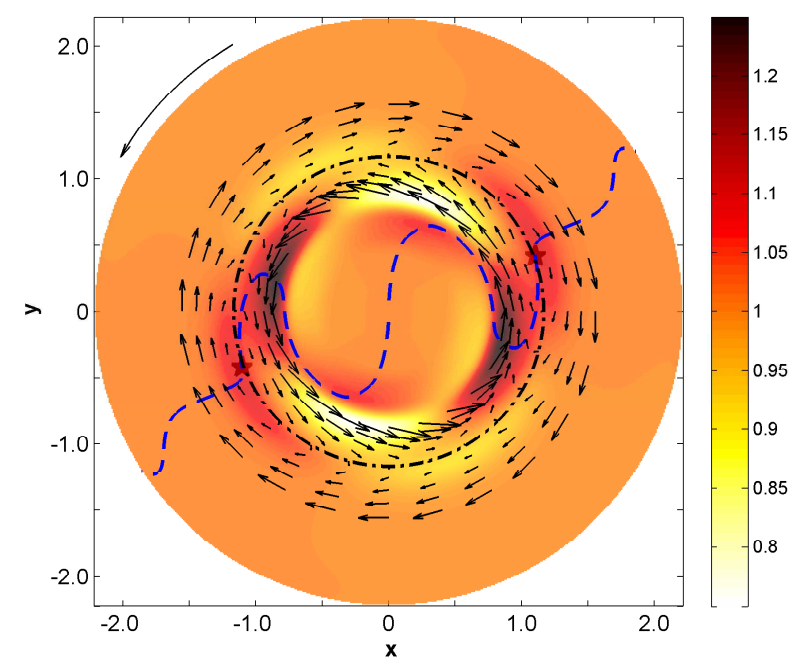

Figure 6. The same eigenfunction $\sigma_{1}(r, \varphi)$, as on Figure 5 , in usual representation. The corotation circle is indicated by a bold black dot-dashed line, the phase curve - by blue dashed line. The arrows represent the perturbed vector velocity field in the coordinate frame of the wave, red stars denote centers of anti-cyclones, large arrow to the north-west indicating the direction of the disk rotation. The outer part of the disk is cut off, where the perturbation amplitude decreases quite rapidly.

As we shall see below, perturbations for the centrifugal branch $C F$ have, in the opposite way, an external wave zone, and it's corotation radius is located slightly inward from the center's radius of the velocity jump.

\section{Influence of gradients of unperturbed surface density of a gas galactic disk on unstable HD modes}

According to observations, in many massive spiral galaxies, the equilibrium surface density $\sigma_{0}(r)$ can vary quite strongly with the radius, even with smoothing of smallscale inhomogeneities.

In the main part of the gas disk, where a global spiral structure is observed, $\sigma_{0}(r)$ of gas is determined by neutral hydrogen $H I$, and is estimated to be close to values corresponding to the boundary of the disk's gravitational stability (Zasov et al. 2011):

$$
\sigma_{0}\left(r \gtrsim 1.5 R_{\alpha \Omega}\right) \approx(5-8) M_{\odot} /(p c)^{2} .
$$

In the inner region of such galaxies, when $r \lesssim(1-1.5) R_{\alpha \Omega}$, there is sometimes a fairly massive disk of molecular hydrogen $\mathrm{H}_{2}$, whose surface density can reach a hundred or more units of $M_{\odot} /(p c)^{2}$, see, for example (Helfer et al. 2003).

In spiral galaxies where there is no such molecular disk at the time of observation, but there is a noticeable star formation in the inner region of the galaxy, indicating the presence of a young stellar pseudo-bulge, we can assume the existence of such a massive gas disk in the recent past.

Therefore, it seems important to investigate the influence of gradients of the unperturbed equilibrium surface density of a gas galactic disk on the development of unstable HD modes in it. The system of equations (3), (4) was used to study unstable HD modes in a gas galactic disk with an inhomogeneous equilibrium surface gas density $\sigma_{0}(r)$. The model density was specified using the function:

$$
\begin{aligned}
\sigma_{0}(r) & =\sigma_{02}\left[\frac{\left(Q_{\sigma}+1\right)}{2}\right. \\
& \left.-\frac{\left(Q_{\sigma}-1\right)}{2} \tanh \left(\frac{r-R_{\sigma}}{L_{\sigma}}\right)\right],
\end{aligned}
$$

where $Q_{\sigma}=\sigma_{01} / \sigma_{02}, \sigma_{01}$ and $\sigma_{02}$ - are the surface densities in the center of the disk and at its periphery, respectively; $R_{\sigma}$ - radius of the density jump center, $L_{\sigma}$ - semithickness of the transition region.

The results of the investigations of unstable HD modes presented below are obtained for a disk with a model profile $\Omega_{\text {th tail }}(r)$, see (7), (8), with parameters given in (10), and with the model function $\sigma_{0}(r)$, defined by the formula (13), with the following set of parameters:

$$
\begin{aligned}
Q_{\sigma} & =20 ; \quad L_{\sigma}=0.1 ; \\
\sigma_{02} & =5 M_{\odot} /(p c)^{2} \quad R_{\sigma} \in[0.4-1.4] ;
\end{aligned}
$$

Such parameters qualitatively correspond to the distributions of the equilibrium surface density of the gas disk, which are observed in some massive spiral galaxies.

It can be assumed that the $\sigma_{0}(r)$ density jump, described by (13), (14), will not be too "narrow". Taking into account the normalization of the linear units over $R_{\alpha \Omega}$, see (10), the chosen width of the $\sigma_{0}$-jump region will be $\sim 2 L_{\sigma} \approx 570 p c$, which is several times larger than the characteristic thickness of the gas disk in its central region.

It was found that the presence of a strong inhomogeneity of the surface density, described by formulas (13), (14), significantly changes the properties of unstable HD modes. In this case, depending on the position $R_{\sigma}$ of the center of the density jump (relative to the center of the velocity jump), individual branches of oscillations can both be weakened, up to stabilization, and appreciably increase in increment, in comparison with a homogeneous disk. 
The results of the simulation performed below are analyzed using calculations for the azimuthal harmonic $m=2$.

We found out that different branches of HD oscillations change in different ways when the inhomogeneity of the surface density is introduced into the model. In particular, the resonant low-frequency branch $A_{1}$ exhibits the local maximums and local minimums of the increment as a function of the changes in the position of the center of the density jump $R_{\sigma}$, with the fixed remaining parameters of the model disk. These changes of the eigenvalue $\omega$ for a typical value $M_{\star}=15$, are shown on Figure 7 .
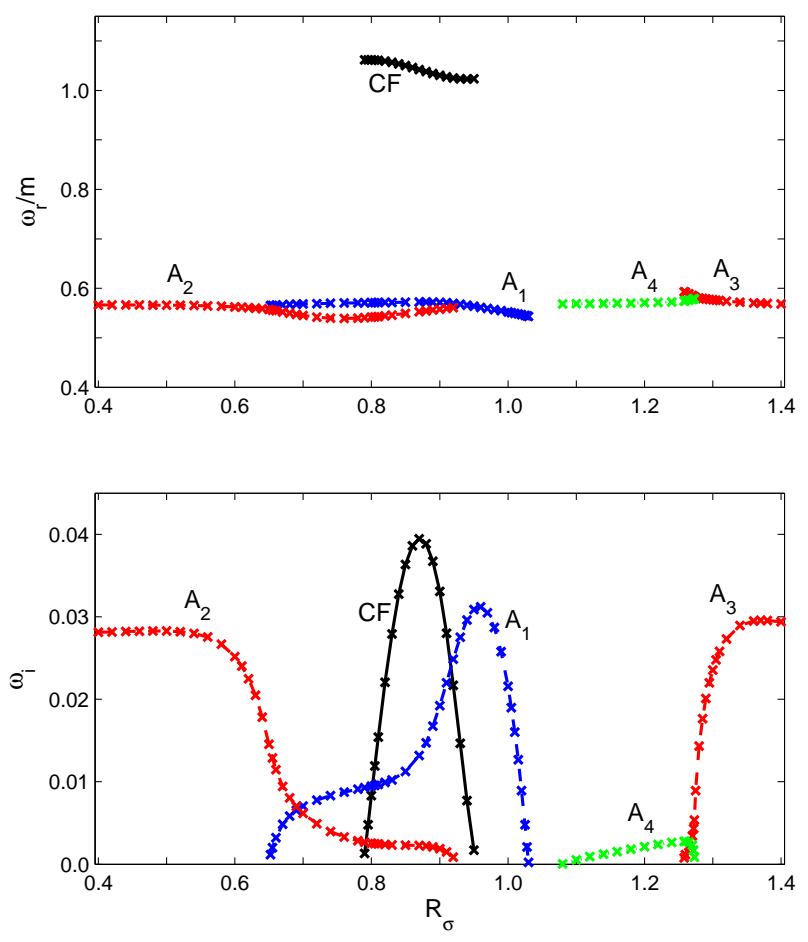

Figure 7. Calculated eigenvalues of the main unstable branches of the $m=2$ harmonic, depending on the parameter $R_{\sigma}$ - the position of the $\sigma_{0}(r)$-jump (relative to $R_{\alpha \Omega}$ - position of the $\Omega(r)$-jump), at fixed value of parameter $M_{*}=15$. The model gas disk has the velocity profile (7), (8) with parameters (10), and $\alpha_{\Omega \min }=-1.85$. The unperturbed surface density $\sigma_{0}(r)$ is described by (13), (14). Top panel - phase angular velocity of waves $\omega_{r} / m$, bottom panel increment $\omega_{i}$.

Another important effect of the influence of density gradients was the "revival" of the branch $C F$, when the position of the density jump, $R_{\sigma}$, falls into a neighborhood of some resonance value, $R_{\sigma 0}$, see Figure 7 . The increment of the $C F$ branch with $R_{\sigma} \approx R_{\sigma 0}$, exceeds the local maximums of the increments of branch $A_{1}$, in the range of the parameter $10 \leqslant M_{\star} \leqslant 25$. However, when the center $R_{\sigma}$ is
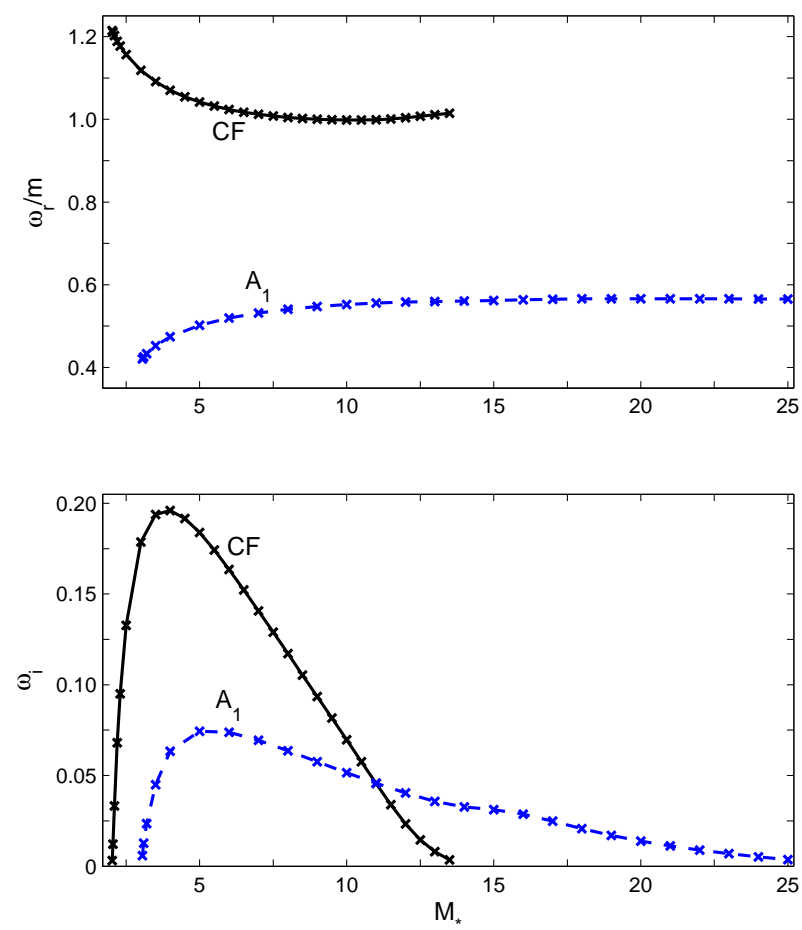

Figure 8. The eigenvalues of the main unstable branches of the $m=2$ harmonic, depending on the parameter $M_{*}$, at fixed value of parameter $R_{\sigma}=0.96$. The model gas disk has the same other parameters, as described in Figure 7.

displaced relative to $R_{\sigma 0}$ by $\gtrsim 10 \%$, the $C F$ branch stabilizes.

We note the well-known property of the growth rate of unstable HD modes, which also manifests itself in the inhomogeneous disk described here. When the parameter $M_{\star}$ increases, starting with the values $M_{\star} \gtrsim(3-5) / \delta V_{\varphi}$, here it means $M_{\star} \gtrsim 10$, with its further growth, the maximum increments of unstable HD modes decrease quite noticeably.

Nevertheless, the magnitudes of the increments near their maxima for the investigated unstable branches of HD modes remains acceptable for the development of these modes in the galactic disk - up to values of the parameter $M_{\star} \approx 20$, see Figure 8 .

Now we can compare, how change the behavior of the perturbations for the unstable $C F$ branch of $\mathrm{HD}$ modes, when we proceed from the model disk with a uniform equilibrium surface density (and the velocity profile steepness parameter $\alpha_{\Omega \text { min }}=-2.0$ ), - to the disk with the $\sigma_{0}$-jumped surface density (and the parameter $\alpha_{\Omega \min }=-1.85$ ).

For comparison, Figures 9 and 10 present the calculated patterns of the perturbed surface density (eigenfunc- 
tion) for an unstable branch $C F$, the harmonic $m=2$, for a parameter value $M_{\star}=15$, for two cases:

on Figure 9 - for a disk with a rotation profile (7), (8) with parameters (10), but with $\alpha_{\Omega} \min =-2.0$, and a uniform equilibrium surface density;

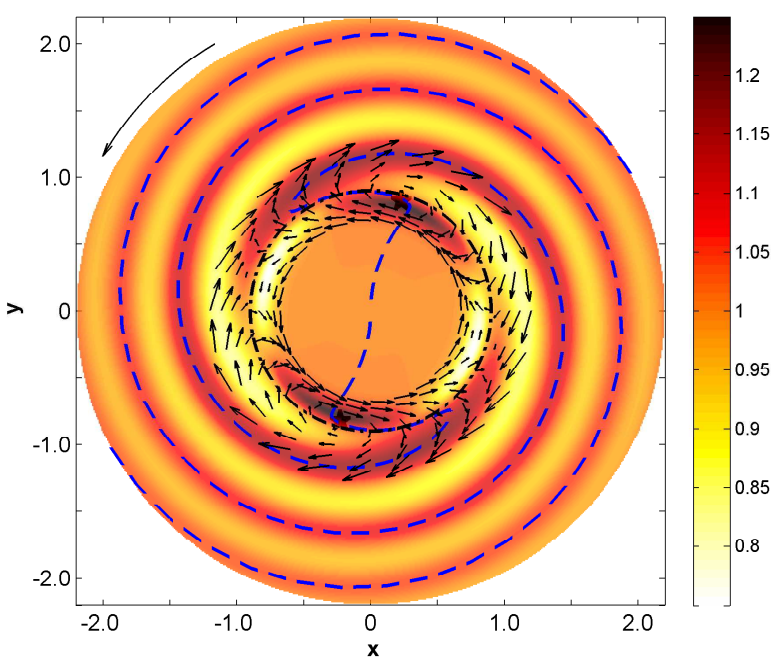

Figure 9. The perturbed surface density $\sigma_{1}(r, \varphi)$ for the unstable branch $C F$, the azimuthal harmonic $m=2$, the parameter $M_{*}=15$. Case of the model disk with a uniform unperturbed surface density, and angular velocity profile (7), (8) with parameters (10), but with $\alpha_{\Omega \min }=-2.0$.

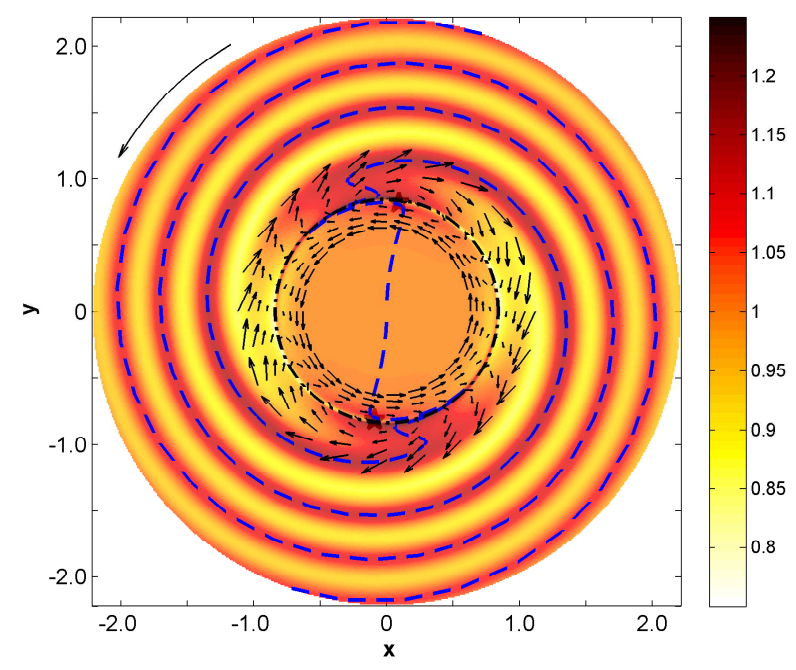

Figure 10. Normalized perturbed surface density $\sigma_{1}(r, \varphi) / \sigma_{0}(r)$, for the unstable branch $C F$, the azimuthal harmonic $m=2$, and parameter $M_{*}=15$. Case of the model disk having an angular velocity profile (7), (8) with parameters (10), parameter $\alpha_{\Omega \text { min }}=-1.85$. The unperturbed surface density $\sigma_{0}(r)$ is described by (13), (14). The scale of the exponential decay of the amplitude with radius, $L_{e}$, for $r>(1.5-2)$ is $L_{e} \approx(3.9-4.0) \mathrm{kpc}$. and on Figure 10 - for a disk with a similar rotation profile, with $\alpha_{\Omega \text { min }}=-1.85$, but with non-uniform equilibrium surface density (13), with parameters (14), and $R_{\sigma}=0.87$.

Note, that the inner part of the perturbations $\sigma_{1}(r, \varphi)$ (near the corotation) for the CF branch in Figure $10-$ is visually similar to the pseudo-ring structure in the case of the $A_{1}$ branch in Figure 6.

The eigenfrequencies, corresponding to the calculated eigenfunctions, are present on the Figure 3 and Figure 7, respectively.

For a disk with an inhomogeneous unperturbed surface density $\sigma_{0}(r)$, described by (13), (14), the distribution of the normalized perturbed surface density $\sigma_{1}(r, \varphi) / \sigma_{0}(r)$ is represented in the Figure 10.

It is this function that actually enters the equations describing the dynamics of small perturbations, see (3), (4). As we see, the behavior of the disturbances on the Figs. 9 and 10 - looks pretty close.

On the other hand, in the case of the $\sigma_{0}(r)$, described by (13) and (14), the density perturbations $\sigma_{1}$ will have a small absolute amplitude in the outer region of the disk (with respect to the density jump position).

Nevertheless, from Figure 10 it can be seen, that the CF branch is able, in principle, to form a regular spiral structure in the main part of the gas galactic disk (in the outer region of the disc relative to the velocity jump).

To show explicitly the possibility of increasing the increments of the CF branch due to the presence of the $\sigma_{0}(r)$ jump, Figure 11 shows the calculated eigenvalues of the main (in increments) unstable branches, depending on the position $R_{\sigma}$ of the center of the density jump for the disk with the profile (10), but with $\alpha_{\Omega \text { min }}=-2.0$, and model $\sigma_{0}(r)$ in the form (13). We see that the presence of a density jump can increase the growth rate of unstable HD branches. In particular, the increment of the CF branch can increase almost twofold in comparison with a disk of a uniform density.

As follows from the results shown in Figures 7 and 8 , the unstable HD modes, which are found in a model disk that is inhomogeneous in the unperturbed surface density, see (13), (14), and have a realistic velocity profile (7), (8) with the velocity profile steepness parameter $\alpha_{\Omega \text { min }}=-1.85$, - quite "weak", i.e. have relatively small increments.

Will such HD modes appear in the gas disk of a real galaxy? The answer is given by an estimate of the characteristic growth time of the perturbations. We use the applied normalization. Taking into account the parameters (10), the normalizing frequency $\Omega_{n o r m}=V_{\varphi \max } / R_{\alpha \Omega}$ 

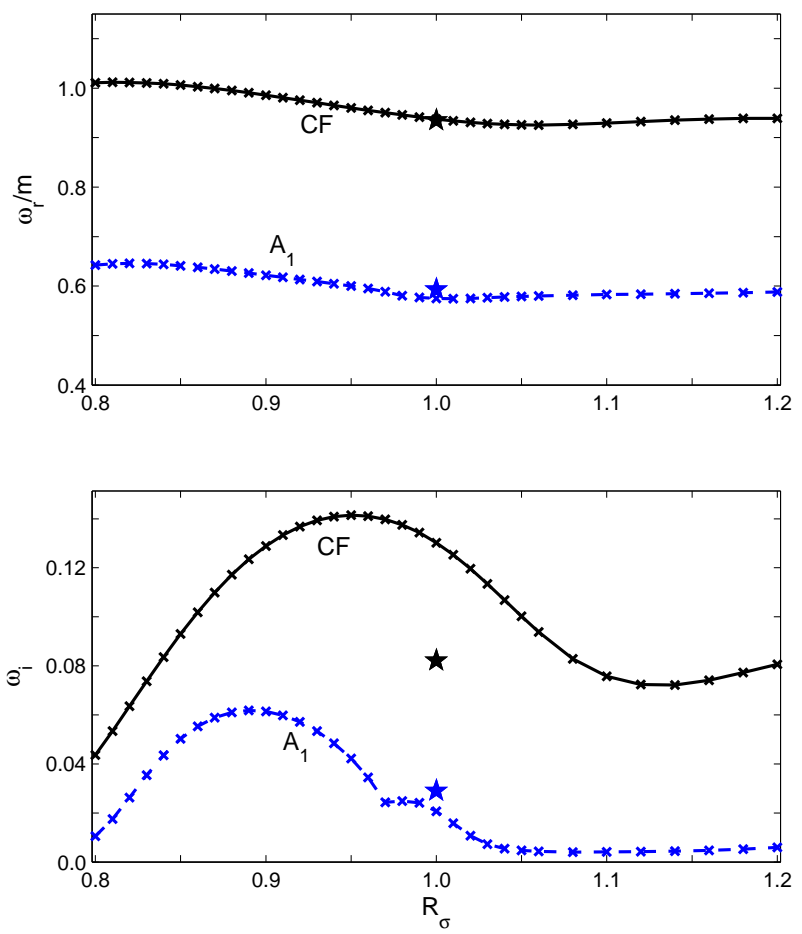

Figure 11. Calculated eigenvalues of the main (in increments) unstable branches of the $m=2$ harmonic, depending on the parameter $R_{\sigma}$, at fixed value of parameter $M_{*}=15$. The model gas disk has the velocity profile (7), (8) with parameters (10), and $\alpha_{\Omega \text { min }}=-2.0$. The unperturbed surface density $\sigma_{0}(r)$ is described by (13), (14). Notation - as in Figure 7. The asterisks show the eigenvalues for the case $\sigma_{0}=$ const. Top panel - phase angular velocity of waves $\omega_{r} / m$, bottom panel - increment $\omega_{i}$.

is: $\Omega_{\text {norm }} \approx 119.3(\mathrm{~km} / \mathrm{s}) / \mathrm{kpc}$. Then, for the time $\tau_{e}$ of the exponential growth of the perturbation amplitude we obtain an approximate formula that is applicable when using a particular set of parameters (10):

$$
\tau_{e}=\frac{[\mathrm{kpc} /(\mathrm{km} / \mathrm{s})]}{\left[\omega_{i} \cdot \Omega_{\text {norm }}\right]} \approx \frac{8.196 \cdot 10^{-3}}{\omega_{i}} \mathrm{GY}
$$

As follows from the calculated eigenvalues of unstable HD modes in the inhomogeneous model gas disk, with the rotation profile (7), (8) and the parameters (10), in which $\sigma_{0}(r)$ is described by formulas (13), (14), the increment of the modes at a typical value of the parameter $M_{\star}=15$, have a magnitude $\omega_{i} \gtrsim 0.03$, see Figure 7 .

Using (15), we obtain an estimate of the time of amplitude growth by a factor of $e: \tau_{e} \lesssim 0.273 G Y$. In this case, the amplitude growth time by an order of magnitude will be: $\tau_{10} \lesssim 1.0 \mathrm{GY}$.

If the values of the parameter $M \star$ in the disk are closer to $M_{\star}=20$, then the increments of the modes, as can be seen from Figure 8, will be almost two times lower than in the case of $M_{\star}=15$, and the corresponding growth times of the amplitude will be as many times as large.

This indicates the weakness of the investigated modes in the model gas disk with the fairly realistic sets of parameters that we use. Moreover, the possibility of generating of an unstable $C F$ branch of perturbations in the "main" range of the parameter $M_{\star}, 15 \lesssim M_{\star} \lesssim 25$, may not be realized in the disk, because of the sensitivity of this branch to the position $R_{\sigma}$, see Figure 7 .

On the other hand, low-frequency branch $A_{1}$ is quite capable of developing in a real disk, because it is preserved in a homogeneous disk, see Figure 4, and in a disk with a nonuniform unperturbed gas surface density, see Figure 7. The values of the increments for this branch of oscillations are satisfactory - up to the values of the parameter $M \star \approx 20$. Such an unstable HD oscillation mode can, under certain conditions, form a large-scale pseudo-ring structure in the gas disk in the region of the velocity jump in the galaxy. If unperturbed surface density of the gas disk in the region of formation of the pseudo-ring was sufficiently large, then, owing to star formation, a similar pseudo-ring will appear in the young stellar disk.

\section{Conclusions}

A study is made of unstable HD modes in a thin, non-selfgravitating model gas disk that approximates the gas disk of a real galaxy whose rotation curve has a velocity jump in the inner region of the disk.

It is shown that in this model disk two different branches of unstable HD modes can be generated the "traditional" $C F$ branch of oscillations, and the lowfrequency branch $A_{1}$. Both branches have rather small increments, at typical values of the temperature parameter $M_{\star}$ in the disk, the times $\tau_{10}$ of grows of the perturbation amplitude by an order of magnitude turn out to be $\tau_{10} \lesssim(1-2) G Y$.

Branch $A_{1}$ has more chances for its manifestation in a real gas galactic disk, because it can be excited both in a uniform disk and in a disk that is inhomogeneous in density. This branch leads to the formation in the vicinity of its corotation radius (the latter lies inside the velocity jump region) of the pseudo-ring in the surface density field.

In the case of a two-arm azimuthal harmonic, these are two tightly wound quarter-turn spirals. If the surface density in the gas disk in the region of formation of this pseudo-ring proves to be high enough, then, due to star formation, a similar pseudo-ring will be formed in the young stellar disk. 
An unstable branch $C F$ generates a regular spiral density wave in the region of the disk behind the velocity jump. However, there are fewer chances for this branch of oscillations, since for its excitation it is necessary that an appreciable density jump also exists in the gas disk, and the center of this jump must fall into a narrow neighborhood of some resonant position.

According to preliminary calculations, at some intervals of the parameter $M *$ a branches of other large scale azimuthal harmonics $-m=1$, and $m=3$ - can be generated with increments, comparable with the ones of the main branches of the $m=2$ harmonic.

Acknowledgment: We are grateful to A.V.Zasov for helpful critical discussions. This research was supported in part by Department of Physical Sciences of RAS, subprogram 'Interstellar and intergalactic media: active and elongated objects', and also by the Russian Basic Research Foundation, grant 16-02-00649.

\section{References}

Arakcheev, A.S. \& Torgashin, Yu.M. 2012, In: Problems of Basic and Applied Natural and Engineering Sciences in the Modern Informational Society (Moscow Institute of Physics and Technology, Moscow), 78-79 (in Russian).

Athanassoula, E. 1992, MNRAS, 259, 345-364.

Bertin, G. 2014, Dynamics of Galaxies, Cambridge University Press, Cambridge.

Binney, J., Tremaine, S. 2008, Galactic Dynamics, Second Edition, Princeton University Press, Princeton.

Bournaud, F., Combes, F., \& Semelin, B. 2005, MNRAS, 364, L18L22.

Chemin, L., Carignan, C., \& Foster, T. 2009, ApJ, 705, 1395-1415.

Combes, F. 2007, In: Combes F., Palous J. (Eds.), Proceedings of the IAUS 235, Cambridge University Press, Cambridge, 19-23.

Einasto, J. \& Haud, U. 1989, A\&A, 223, 89-94.

Freeman, K.C. 2009, Proceedings of the Astronomical Society of the Pacific conference, ASP, San Francisco, 3-11.

Fridman, A.M. 1990, Sov. Phys. JETP, 71, 627-635.

Fridman, A.M., Polyachenko, E.V., Torgashin, Yu.M., Yanchenko, S.G.,\& Snezhkin, E.N. 2006, Physics Letters A, 349, 198-211.
Fridman, A.M. \& Polyachenko, V.L. 1984, Physics of gravitating systems, I. Equilibrium and stability, Springer Verlag, New YorkBerlin-Heidelberg-Tokyo.

Fridman, A.M., Snezhkina, E.N., Chernikov, G.P., Rylov, A.Yu., Titishova, K.B., \& Torgashin, Yu.M. 2008, Physics Letters A, 372, 4822-4826.

Goldreich, P., Lynden-Bell, D. 1965, MNRAS, 130, 97-124.

Haan, S. et al. 2009, AAS, 41, 747.

Helfer, T.T., Thornley, M. D., Regan, M. W., Wong, T., Sheth, K., Vogel, S. N. et al. 2003, ApJS, 145, 2, 259-327.

Holmberg, J., Nordström, B., Andersen, J. 2007, A\&A, 475, 519-537.

Kolykhalov, P.I. 1984, Instability of a shear discontinuity in a gas moving near a wall, In: Fluid Dynamics (Historical Archive), 19, 465-469.

Kondratyev, B.P. 2014, MNRAS, 442, 1755-1766.

Kormendy, J. 2013, In: Falcon-Barroso J., Knapen J.H. (Eds.), Secular evolution of galaxies, Cambridge University Press, Cambridge, $1-159$.

Kormendy, J. \& Kennicutt, R.C., Jr. 2004, ARA\&A, 42, 603-683.

Lin, C.C. \& Shu, F.H. 1964, ApJ, 140, 646-655.

Lin, C.C. \& Shu, F.H. 1966, PNAS, 55, 229-234.

Morozov, A.G. 1977, Soviet Astronomy Letters, 3, 103-105.

Morozov, A.G. 1979, Soviet Astronomy, 23, 278-281.

Morozov, A.G., Nezlin, M.V., Snezhkin, E.N., Torgashin, Yu.M., \& Fridman, A.M. 1985, Astron. Tsirk. (in Russian), 1414, 4-7.

Morozov, A.G., Nezlin, M.V., Snezhkin, E.N., Torgashin, Yu.M., \& Fridman, A.M. 1985, Astron. Tsirk., 1414, 7-8 (in Russian).

Polyachenko, E.V. 2004, MNRAS, 348, 345-354.

Safronov, V.S. 1960, AnAp, 23, 979-982.

Salo, H., Rautiainen, P., Buta, R., Purcell, G. B., Cobb, M. L., Crocker, D. A. et al. 1999, AJ, 117, 792-810.

Sofue, Y. \& Rubin, V. 2001, ARA\&A, 39, 137-174.

Sellwood, J.A. 2011, MNRAS, 410, 1637-1646.

Tenjes, P., Haud, U., Einasto, J. 1998, A\&A, 335, 449-462.

Toomre, A. 1964, ApJ, 139, 1217-1238.

Toomre, A. 1981, In: Fall S.M., Lynden-Bell D. (Eds.), Proceedings of the Advanced Study Institute, Cambridge University Press, Cambridge \& New York, 111-136.

Torgashin, Yu.M. 1986, The collective processes in gaseous galactic and accretion disks, Ph.D. thesis, Tartu.

Torgashin, Yu.M. \& Omurkanov, T.Z. 2013, AN, 334, 870.

van der Kruit, P.C., \& Freeman, K.C. 2011, $A R A \& A, 49,301-371$.

Weinzirl, T., Jogee, S., Khochfar, S., Burkert, A., \& Kormendy, J. 2009, ApJ, 696, 411-447.

Zasov, A.V., Khoperskov, A.V., \& Saburova, A.S. 2011, Astron Letters (in Russian), 37, 374-384.

Zasov, A.V. \& Sil'chenko, O.K. 2010, Uspekhi Fizicheskih Nauk (in Russian), 180, 434-439. 